\title{
Combination of rating conflict in failure mode and effects analysis based on generalized combination rule
}

\author{
Xuelian Zhou ${ }^{1, \text { a }}$, Feng Liu, ${ }^{1, b}$ and Yongchuan Tang ${ }^{1, c}$ \\ ${ }^{1}$ School of Computer and Information Science, Southwest University, Chongqing 400715, China \\ axlz@swu.edu.cn, bliuf@swu.edu.cn, ctangyc@swu.edu.cn
}

Keywords: Failure mode and effects analysis (FMEA), generalized evidence theory (GET), generalized combination rule (GCR), risk priority number (RPN), conflict combination

Abstract. Failure mode and effects analysis (FMEA) is a preventive tool to keep the bad product from going to the consumers. Experts in a FMEA group often have different evaluations to each failure mode, and this will lead to conflict ratings for each failure mode, then how to manage the conflict to get the risk priority number (RPN) more reasonable is still an open issue. In this paper, the generalized combination rule (GCR) in generalized evidence theory (GET) is introduced to manage the conflicts from different experts in a FMEA group. And the conflicts are modeled as the empty set, which corresponds to the incomplete knowledge or experience among the experts in the FMEA group. In this way, this paper provides a new FMEA model to combine the rating conflict. A real case study to the rotor blades of an aircraft turbine shows the effectiveness and merits of the proposed approach.

\section{Introduction}

Failure mode and effects analysis (FMEA)[1] is quite an important tool for defining, identifying and preventing the known or unknown potential problem of a system, design, service, process and so on, and it has been widely used in many fields since it was built in 1960s[2-8]. At the same time, some of the potential problems in the conventional FMEA model are criticized [9], and a lot of approaches or modified FMEA models are presented in the literatures [10-14]. Among all these shortcomings in the conventional FMEA model, the three risk factors are difficult to be precisely evaluated can be addressed by Dempster-Shafer evidence theory [15-17], and there are already some research achievements among the literatures $[2-4,10,13]$ which are based on the evidence theory.

Lately, some new theories based on the Dempster-Shafer evidence theory are proposed [18-22] and there seems to be some new merits compared with the conventional evidence theory. One of them is the generalized evidence theory (GET)[18-20]. And the generalized combination rule (GCR) in GET can combine the conflict information more effectively and accurately. So, in this paper, the GCR in GET is used as a new approach to deal with the evaluation information among all the experts in the FMEA group.

The rest of this paper is organized as follows. In Section 2, the risk priority number (RPN) and the GCR in GET are introduced briefly. In Section 3, the proposed FMEA model for rating conflict combination based on GCR in GET is presented, then the proposed conflict free RPN (CFRPN) is used to handle a real FMEA case of rotor blades for an aircraft turbine in Section 4. The conclusions are presented in the final Section 5.

\section{Preliminaries}

In this section, the risk priority number (RPN) in failure mode and effects analysis (FMEA) and the generalized combination rule (GCR) in generalized evidence theory (GET) are introduced briefly.

\section{Risk priority number}

One of the most important roles for a FMEA file is to provide the priority among all the potential failure modes by RPNs, and in this way, the limited resource can be used to handle those failure modes with a higher risk level. In conventional FMEA, the RPN is the product of the rating of three risk factors, as 
shown in Eq. (1). In detail, these three risk factors are the probability of the occurrence of a failure mode $(\mathrm{O})$, the severity of a failure effect (S) and the probability of a failure being detected (D) [3, 23].

$\mathrm{RPN}=\mathrm{O} \times \mathrm{S} \times \mathrm{D}$.

For each risk factor, the numerical ratings are from 1 to 10 , the suggested criteria for the risk factor $\mathrm{O}, \mathrm{S}$ and $\mathrm{D}$ are shown in Table 1, Table 2 and Table 3 respectively $[3,23]$.

Table 1 Suggested criteria of rating for occurrence of a failure in FMEA

\begin{tabular}{lll}
\hline Rating & Probability of occurrence & Possible failure rate \\
\hline 10 & Extremely high: failure almost inevitable & $\geq 1 / 2$ \\
9 & Very high & $1 / 3$ \\
8 & Repeated failures & $1 / 8$ \\
7 & High & $1 / 20$ \\
6 & Moderately high & $1 / 80$ \\
5 & Moderate & $1 / 400$ \\
4 & Relatively low & $1 / 2000$ \\
3 & Low & $1 / 15000$ \\
2 & Remote & $1 / 150000$ \\
1 & Nearly impossible & $\leq 1 / 1500000$ \\
\hline
\end{tabular}

Table 2 Suggested criteria of rating for severity of a failure in FMEA

\begin{tabular}{|c|c|c|}
\hline Rating & Effect & Criteria: Severity of effect \\
\hline 10 & $\begin{array}{l}\text { Hazardous without } \\
\text { warning }\end{array}$ & $\begin{array}{l}\text { Failure is hazardous, and occurs without warning. It suspends operation of the system } \\
\text { and/or involves noncompliance with government regulations }\end{array}$ \\
\hline 9 & $\begin{array}{l}\text { Hazardous with } \\
\text { warning }\end{array}$ & $\begin{array}{l}\text { Failure involves hazardous outcomes and/or noncompliance with government } \\
\text { regulations or standards }\end{array}$ \\
\hline 8 & Extreme & Product is inoperable with loss of primary function. The system is inoperable \\
\hline 7 & Major & Product performance is severely affected but functions. The system may not operate \\
\hline 6 & Significant & Product performance is degraded. Comfort or convince functions may not operate \\
\hline 5 & Moderate & Moderate effect on product performance. The product requires repair \\
\hline 4 & Low & Small effect on product performance. he product does not require repair \\
\hline 3 & Minor & Minor effect on product or system performance \\
\hline 2 & Very minor & Very minor effect on product or system performance \\
\hline 1 & None & No effect \\
\hline
\end{tabular}


Table 3 Suggested criteria of rating for detection of a failure in FMEA

\begin{tabular}{|c|c|c|}
\hline Rating & Detection & Criteria: likelihood of detection by design control \\
\hline 10 & $\begin{array}{l}\text { Absolute } \\
\text { uncertainty }\end{array}$ & $\begin{array}{l}\text { Design control does not detect a potential cause of failure or subsequent failure mode; or } \\
\text { there is no design control }\end{array}$ \\
\hline 9 & Very remote & $\begin{array}{l}\text { Very remote chance the design control will detect a potential cause of failure or } \\
\text { subsequent failure mode }\end{array}$ \\
\hline 8 & Remote & $\begin{array}{l}\text { Remote chance the design control will detect a potential cause of failure or subsequent } \\
\text { failure mode }\end{array}$ \\
\hline 7 & Very low & $\begin{array}{l}\text { Very low chance the design control will detect a potential cause of failure or subsequent } \\
\text { failure mode }\end{array}$ \\
\hline 6 & Low & $\begin{array}{l}\text { Low chance the design control will detect a potential cause of failure or subsequent } \\
\text { failure mode }\end{array}$ \\
\hline 5 & Moderate & $\begin{array}{l}\text { Moderate chance the design control will detect a potential cause of failure or subsequent } \\
\text { failure mode }\end{array}$ \\
\hline 4 & Moderately high & $\begin{array}{l}\text { Moderately high chance the design control will detect a potential cause of failure or } \\
\text { subsequent failure mode }\end{array}$ \\
\hline 3 & High & $\begin{array}{l}\text { High chance the design control will detect a potential cause of failure or subsequent } \\
\text { failure mode }\end{array}$ \\
\hline 2 & Very high & $\begin{array}{l}\text { Very high chance the design control will detect a potential cause of failure or subsequent } \\
\text { failure mode }\end{array}$ \\
\hline 1 & Almost certain & $\begin{array}{l}\text { Design control will almost certainly detect a potential cause of failure or subsequent } \\
\text { failure mode }\end{array}$ \\
\hline
\end{tabular}

\section{Generalized combination rule}

In GET [18], the generalized basic probability assignment (GBPA) corresponds to basic probability assignment (BPA) in Dempster-Shafer evidence theory, which is used for data expression and modeling. And the generalized combination rule (GCR) is provided for combining the different evidence.

Suppose that $U$ is a frame of discernment, for a proposition $A, \forall A \subset U$, a mass function is a mapping $m: 2^{U} \rightarrow[0,1]$ that satisfies:

$$
\sum_{A \in 2^{U}} m(A)=1 \text {. }
$$

Where $m$ is the GBPA in the frame of discernment, $U$. Note that, compared to Dempster-Shafer evidence theory, $m(\phi)=0$ is not necessary in GBPA. And it can be used to model other uncertain factor in the open world.

The GCR for two GBPAs, $m_{1}$ and $m_{2}$, is defined as follows [18, 19], from Eq. (3) to Eq. (6).

$$
\begin{aligned}
& m(A)=\frac{(1-m(\phi)) \sum_{B \cap C=A} m_{1}(B) \cdot m_{2}(C)}{1-K}, \\
& K=\sum_{B \cap C=\varnothing} m_{1}(B) \cdot m_{2}(C),
\end{aligned}
$$




$$
\begin{aligned}
& m(\phi)=m_{1}(\phi) \cdot m_{2}(\phi), \\
& m(\varnothing)=1 \text { if and only if } K=1 .
\end{aligned}
$$

Where $m(\phi)=m_{1}(\phi) \cdot m_{2}(\phi)$, it means that the intersection between two empty sets is still an empty set, and this can be explained as the unknown situation is still uncertain.

\section{Rating conflict combination in failure mode and effects analysis}

In this section, the source of the rating conflicts in failure mode and effects analysis (FMEA) are analyzed firstly; then with generalized combination rule (GCR) in generalized evidence theory (GET), the rating conflicts from different experts in a FMEA group are modeled properly; finally, the modified risk priority number (RPN) of each failure mode, which will be a conflict free RPN, noted as CFRPN, is presented.

\section{Rating conflict analysis in FMEA}

The rating conflict for each failure mode comes from different experts in a FMEA group, and the possible reasons of different ratings given by different team member are mainly summarized as follows.

- Different perspectives because of different roles and different responsibilities in a company or other organizations. Each expert may come from different department of an organization, e.g. the Research \& Development department, the Production department, the Experiment department, the Market department, the Sales department and so on; thus each FMEA group member assesses the same failure mode from different perspectives.

- Different academic backgrounds among all the team members. The team members may have different majors before they leave the college or university.

- Different experience. Different work experience and living experience can have effects on their subjective assessment on the same things, especially the professional work experience.

According to these reasons mentioned above, the rating conflict seems to be a kind of knowledge or experience conflict. And it can be represented as the empty set in GET, which means the knowledge conflict among team members. With GCR in GET, the quantitative rating conflict among the experts is combined in the following sections.

\section{Quantification of rating conflict}

In real application, an expert in a FMEA group may assess the rating level to more than one level with percentages distribution[2,3], and in this way, the total percentage of one expert for one failure mode will be no more than $100 \%$. What's more, the rating level given by different experts in a FMEA group sometimes is quite different with each other. At this time, a conflict among different experts in a FMEA group happens. This is reasonable, because each team member has one's own judgment basing on one's own knowledge and experience comes from different academic background, work experience, living experience and so on. And these difference contribute to each expert's special skill and knowledge as well as one's limitation, and this the limitation can limit an experts' judgment, this can be regarded as the incomplete frame of discernment of each expert in GET, and it is out of the 10 ratings. Here, in this paper, the degree of two experts' conflict because of the incomplete knowledge is represented by $m(\phi)$ in GET.

Definition 1 The degree of the conflict $m(\phi)$ between two experts for a particular failure mode, is one tenth of the average value of the difference of the maximal ratings between these two experts plus the difference of the minimal ratings, as shown in Eq.(7).

$$
m(\phi)=\frac{\frac{1}{2}\left(\left|m_{\max }^{E_{1}}-m_{\max }^{E_{2}}\right|+\left|m_{\min }^{E_{1}}-m_{\min }^{E_{2}}\right|\right)}{10}
$$

The Eq. (7) replaces the Eq. (5) and Eq. (6) in GET. And in function (7), $m_{\max }^{E_{1}}$ and $m_{\max }^{E_{2}}$ represent the maximum rating evaluated by two experts, $E_{1}$ and $E_{2}$, while $m_{\min }^{E_{1}}$ and $m_{\text {min }}^{E_{2}}$ represent the 
minimum one. Sometimes, the maximum one equals the minimum one, e.g. the expert assesses a single rating with a belief of $100 \%$.

As to Eq. (7), if a team member assigns an empty set to nonzero, in other words, if one keeps in mind that one's knowledge or frame of discernment is incomplete, the empty set in Eq. (7) can be degenerated to its original type, the Eq.(5) and Eq. (6).

According to Eq. (7), some numerical characteristics and properties of the conflict can be summarized as follows.

(1) The minimum of $m(\phi)$ is zero. When $m(\phi)=0$, it means that both experts rate a particular failure mode among the same interval, with the same or different probability in the interval.

(2) The maximum of $m(\phi)$ is 0.9 , which means the biggest conflict between two experts, and one expert has a total belief that the level is 10 and the other expert think it should be 1 .

(3) The interval of $m(\phi)$ is from 0 to 0.9 .

(4) If and only if $m(\phi)=0$, the GCR in GET used by the proposed method in this paper degenerates to the conventional Dempster combination rule in Dempster-Shafer evidence theory.

\section{Conflict free RPN}

Assume that there are $L$ experts $\left(E_{1}, E_{2}, \ldots, E_{L-1}, E_{L}\right)$ in a FMEA group, and there are $N$ failure modes $\left(F_{1}, F_{2}, \ldots, F_{N-1}, F_{N}\right)$ needs to be taken into consideration. As mentioned before, the rating conflict among the experts comes from the incomplete knowledge or experience of each team member. Thus the incomplete frame of discernment, $\theta_{i}{ }^{n}$, of the $n$th failure mode to the $i$ th risk factor can be expressed as Eq. (8).

$$
\theta_{i}^{n}=(1,2,3,4,5,6,7,8,9,10) ; i=O, S, D ; n=1,2, \mathrm{~L}, N
$$

Definition 2 The conflict free RPN (CFRPN) for a failure mode can be got according to the following four steps.

Step 1: Construct the belief function of each expert for each failure mode.

The generalized basic probability assignment (GBPA) is similar to the basic probability assignment (BPA) in the literatures[15-17], and it is denoted as function $m(A)$, and it is a mapping: $m(A): 2^{\theta} \rightarrow[0,1]$. The difference from literature is the assignment of the empty set, $m(\phi)$, if it is assigned as zero in GBPA, it will be calculated by Eq. (7) in Section 3.2.

$$
\sum_{A \in 2^{\theta}} m(A)=1
$$

In Eq. (9), $\theta=(1,2,3,4,5,6,7,8,9,10), m(A)$ expresses the probability given by experts to support proposition $A$, which may be no more than a single possible rating of a risk factor for a failure mode, and it may be a set of multiple possible ratings.

Step 2: Calculate the quantitative rating conflict.

If the GBPA for empty set is zero in Step 1, then with Eq. (7), the rating conflict can also be quantified, and sometimes it may also be zero because the conflict is not always exist. And if the initial GBPA given by experts for empty set is not zero, it can be explained as the incomplete or uncertain knowledge of the expert which is also a conflict to the rating.

Step 3: Combine the GBPAs from different experts in the FMEA group.

With the combination by GCR in Eq. (3)- Eq. (6), the conflict free ratings of each risk factor for all the failure modes can be got. What's more, because the GCR in GET is similar to Dempster combination rule, and it is commutative and associative, it can be calculated in any order or at the same time. And for the $n$th failure mode to the $i$ th risk factor, for each rating represented by a proposition $A \subset \theta_{i}^{n}$, the combined probability assignment from the $L$ experts is defined as Eq. (10).

$$
M_{i}^{n}(A)=\left(M_{i 1}^{n} \oplus M_{i 2}^{n} \oplus \mathrm{L} \oplus M_{i L}^{n}\right)(A)
$$

Step 4: Calculate CFRPNs of all the failure modes. 
For the $n$th failure mode, the CFRPN is calculated as Eq. (11). And the conflict mass function, represented by Eq. (7), which means uncertain and conflict, are assigned to the average rating of the whole ratings, and in this paper it is assumed as the rating 6.

$$
C F R P N_{n}=\left[O \cdot M_{O}^{n}(A)+6 \cdot M_{O}^{n}(\phi)\right] \times\left[S \cdot M_{S}^{n}(A)+6 \cdot M_{S}^{n}(\phi)\right] \times\left[D \cdot M_{D}^{n}(A)+6 \cdot M_{D}^{n}(\phi)\right]
$$

The illustrative numerical example and application of these steps can be found in a real case study in the following Section 4.

\section{A real case study}

In this section, the proposed approach is used to handle a real FMEA case of rotor blades for an aircraft turbine[3], and there are 17 typical failure modes among these rotor blades, which will be handled in this section to show the effectiveness of the proposed FMEA model.

\section{Experiment data}

Consider the 17 failure modes assessed by three experts in Table 4 [3], the belief function is constructed by experts according to literature [3].

Table 4 The 17 failure modes evaluated by three experts in literature[3].

\begin{tabular}{|c|c|c|c|c|c|c|c|c|c|}
\hline \multirow{3}{*}{$\begin{array}{c}\text { Failure } \\
\text { mode }\end{array}$} & \multicolumn{9}{|c|}{ Rating of risk factor } \\
\hline & \multicolumn{3}{|c|}{ Expert 1} & \multicolumn{3}{|c|}{ Expert 2} & \multicolumn{3}{|c|}{ Expert 3} \\
\hline & $\mathrm{O}$ & $S$ & $\mathrm{D}$ & $\mathrm{O}$ & $S$ & $\mathrm{D}$ & $\mathrm{O}$ & $S$ & $\mathrm{D}$ \\
\hline \multirow[t]{2}{*}{1} & $3: 40 \%$ & 7 & 2 & $3: 90 \%$ & 7 & 2 & $3: 80 \%$ & 7 & 2 \\
\hline & $4: 60 \%$ & & & $4: 10 \%$ & & & $4: 20 \%$ & & \\
\hline \multirow[t]{2}{*}{2} & 2 & 8 & 4 & 2 & $8: 70 \%$ & 4 & 2 & 8 & 4 \\
\hline & & & & & $9: 30 \%$ & & & & \\
\hline 3 & 1 & 10 & 3 & 1 & 10 & 3 & 1 & 10 & 3 \\
\hline \multirow[t]{2}{*}{4} & 1 & $6: 80 \%$ & 3 & 1 & 6 & 3 & 1 & 6 & 3 \\
\hline & & $7: 20 \%$ & & & & & & & \\
\hline \multirow[t]{2}{*}{5} & 1 & 3 & $2: 50 \%$ & 1 & 3 & $2: 70 \%$ & 1 & $3: 60 \%$ & 1 \\
\hline & & & $1: 50 \%$ & & & $1: 30 \%$ & & $2: 40 \%$ & \\
\hline 6 & 2 & 6 & 5 & 2 & 6 & 5 & 2 & 6 & 5 \\
\hline 7 & 1 & 7 & 3 & 1 & 7 & 3 & 1 & 7 & 3 \\
\hline \multirow[t]{2}{*}{8} & 3 & $5: 60 \%$ & 1 & 3 & $5: 80 \%$ & 1 & 3 & $5: 80 \%$ & 1 \\
\hline & & $6: 40 \%$ & & & $6: 20 \%$ & & & $7: 20 \%$ & \\
\hline \multirow[t]{2}{*}{9} & $2: 90 \%$ & $10: 60 \%$ & 4 & $2: 75 \%$ & $10: 90 \%$ & 4 & $2: 80 \%$ & $10: 90 \%$ & 4 \\
\hline & $1: 10 \%$ & $9: 40 \%$ & & $1: 25 \%$ & $9: 10 \%$ & & $1: 20 \%$ & $9: 10 \%$ & \\
\hline 10 & 1 & 10 & 6 & 1 & 10 & 6 & 1 & 10 & 6 \\
\hline 11 & 1 & 10 & 5 & 1 & 10 & 5 & 1 & 10 & 5 \\
\hline
\end{tabular}




\begin{tabular}{|c|c|c|c|c|c|c|c|c|c|}
\hline 12 & 1 & 10 & $6: 60 \%$ & 1 & 10 & $5: 80 \%$ & 1 & 10 & $6: 70 \%$ \\
\hline & & & $5: 40 \%$ & & & $4: 20 \%$ & & & $5: 30 \%$ \\
\hline 13 & 1 & 10 & $5: 80 \%$ & 1 & 10 & 5 & 1 & 10 & 5 \\
\hline & & & $4: 20 \%$ & & & & & & \\
\hline 14 & 1 & 10 & 6 & 1 & 10 & $6: 80 \%$ & 1 & 10 & 6 \\
\hline & & & & & & $7: 20 \%$ & & & \\
\hline 15 & 2 & $7: 95 \%$ & 3 & 2 & 7 & 3 & 2 & 7 & $3: 70 \%$ \\
\hline & & $6: 5 \%$ & & & & & & & $4: 30 \%$ \\
\hline 16 & $2: 90 \%$ & 4 & 3 & $2: 75 \%$ & 4 & 3 & $2: 80 \%$ & 4 & $3: 80 \%$ \\
\hline & 1:10\% & & & $1: 250 \%$ & & & $1: 20 \%$ & & $2: 20 \%$ \\
\hline 17 & 2 & $5: 90 \%$ & 3 & 2 & $5: 90 \%$ & 3 & 2 & $5: 60 \%$ & 3 \\
\hline & & $6: 10 \%$ & & & $6: 10 \%$ & & & 6:40\% & \\
\hline
\end{tabular}

\section{Experiment with the proposed approach}

The evaluation information assessed by each expert for each risk factor of all these 17 failure modes are shown in Table 4, and it comes from the literature [3]. In this way, the result of the proposed approach can be compared with the data in the literature.

Without losing the generality, take the 12th failure mode as an example to illustrate how the proposed approach is used to calculate the conflict free RPN (CFRPN), as described step by step in Section 3.3.

Step 1: Construct the belief function of each expert for the 12th failure mode.

For the 12th failure mode, the GBPAs for each risk factor are shown as follows.

O: $m_{O 1}^{12}(1)=m_{O 2}^{12}(1)=m_{O 3}^{12}(1)=1.0 . S: m_{S 1}^{12}(10)=m_{S 2}^{12}(10)=m_{S 3}^{12}(10)=1.0$.

$\mathrm{D}: m_{D 1}^{12}(6)=0.6, m_{D 1}^{12}(5)=0.4 ; m_{D 2}^{12}(5)=0.8, m_{D 2}^{12}(4)=0.2 ; m_{D 3}^{12}(6)=0.7, m_{D 3}^{12}(5)=0.3$.

Step 2: Get the quantitative rating conflict for the 12th failure mode.

For the 12th failure mode, the rating conflict exits for risk factor $\mathrm{D}$, and there is no rating conflict for risk factor $\mathrm{O}$ and $\mathrm{S}$. For the risk factor $\mathrm{D}$, the rating conflict between Expert 1 and Expert 2 is calculated according to Eq.(7).

$m_{D(1,2)}^{12}(\phi)=\frac{\frac{1}{2}\left(\left|m_{\max }^{E_{1}}-m_{\max }^{E_{2}}\right|+\left|m_{\text {min }}^{E_{1}}-m_{\text {min }}^{E_{2}}\right|\right)}{10}=\frac{\frac{1}{2} *[(6-5)+(5-4)]}{10}=0.1$.

Step 3: Combine the GBPAs from different experts in the FMEA group for the 12th failure mode.

$K_{D(1,2)}^{12}=m_{D 1}^{12}(6) \cdot\left[m_{D 2}^{12}(5)+m_{D 2}^{12}(4)\right]+m_{D 1}^{12}(5) \cdot m_{D 2}^{12}(4)=0.68$.

$m_{D(1,2)}^{12}(5)=\frac{\left[1-m_{D(1,2)}^{12}(\phi)\right] \cdot\left[m_{D 1}^{12}(5) \cdot m_{D 2}^{12}(5)\right]}{1-K_{D(1,2)}^{12}}=\frac{(1-0.1) \cdot 0.4 \cdot 0.8}{1-0.68}=0.9$.

Repeat the Step 2 and Step 3, and all the GBPAs are got.

$$
\begin{aligned}
& M_{D}^{12}(\phi)=m_{D((1,2), 3)}^{12}(\phi)=0.05, \\
& K_{D}^{12}=K_{D((1,2), 3)}^{12}=0.73,
\end{aligned}
$$


$M_{D}^{12}(5)=m_{D((1,2), 3)}^{12}(5)=0.95$.

And for other two risk factors in the 12th failure mode, the GBPAs are shown as follows.

$M_{O}^{12}(\phi)=0, K_{O}^{12}=0, M_{O}^{12}(1)=1$.

$M_{S}^{12}(\phi)=0, K_{S}^{12}=0, M_{S}^{12}(10)=1$.

Step 4: Calculate the CFRPN of the 12th failure mode.

$$
\begin{aligned}
\text { CFRPN }_{12} & =\left[O \cdot M_{O}^{n}(A)+6 \cdot M_{O}^{n}(\phi)\right] \times\left[S \cdot M_{S}^{n}(A)+6 \cdot M_{S}^{n}(\phi)\right] \times\left[D \cdot M_{D}^{n}(A)+6 \cdot M_{D}^{n}(\phi)\right] \\
& =1 \cdot 1 \times 1 \cdot 10 \times(5 \cdot 0.95+5 \cdot 0.05)=50.5
\end{aligned}
$$

Repeat Step 1 to Step 4, the CFRPNs for all these 17 failure modes(FM) are presented in Table 5, as well as the comparing MVRPNs in the literature[3] where the experiment data comes from.

\begin{tabular}{|c|c|c|c|}
\hline \multicolumn{2}{|c|}{ Component failure mode } & CFRPN & MVRPN \\
\hline \multirow{5}{*}{ 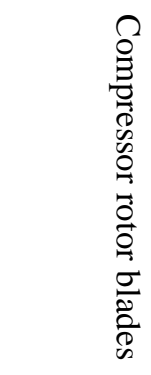 } & FM1 & 42.56 & 42.56 \\
\hline & FM2 & 64 & 64.00 \\
\hline & FM3 & 30 & 30.00 \\
\hline & FM4 & 18 & 18.00 \\
\hline & FM5 & 3.72 & 4.17 \\
\hline \multirow{12}{*}{ 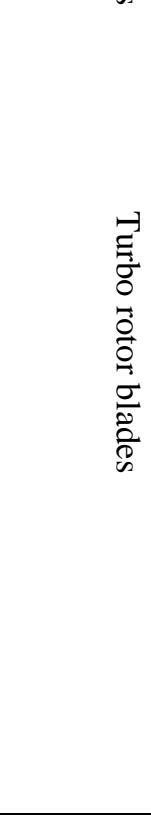 } & FM6 & 60 & 60.00 \\
\hline & FM7 & 21 & 21.00 \\
\hline & FM8 & 15.15 & 15.00 \\
\hline & FM9 & 78.92 & 78.92 \\
\hline & FM10 & 60.00 & 60.00 \\
\hline & FM11 & 50 & 50.00 \\
\hline & FM12 & 50.5 & 50.00 \\
\hline & FM13 & 50 & $60.00^{*}$ \\
\hline & FM14 & 60 & 60.00 \\
\hline & FM15 & 42 & 42.00 \\
\hline & FM16 & 23.88 & 23.88 \\
\hline & FM17 & 30.05 & $50.90^{* *}$ \\
\hline
\end{tabular}

Table 5 The CFRPNs, compared with MVRPNSs in literature[3].

* The value is incorrect in the literature [3], and it should be 50 .

$* *$ The value is incorrect in the literature [3], and it should be 30 .

\section{Results and discussions}

According to Section 4.2, the CFRPNs of the 17 failure modes (FM) are calculated with the proposed FMEA model in Section 3, as shown in Table 5.

In Table 5, the CFRPNs of FM1 and FM9 are the same with the MVRPNs, this is because for these two evaluation information, the GCR in GET is degenerated to the conventional combination rule in conventional D-S Evidence Theory [15-16]; which is a merit of GET. For FM2, FM4, FM13, FM14, FM15, FM16 and FM17, the results are consistent with the MVRPNs, which mean the proposed approach compatibles the old one. For FM3, FM6, FM7, FM10 and FM11, there is no rating conflict according to the experiment data. And according to FM5, the CFRPN is small than MVRPN, and it can be seen as an optimistic strategy, because the rating of the failure mode is reduced according to the proposed approach; What's more, compared with Su's modified method[4], it's more close to the 
original one of Yang's MVRPN, and how to control the RPN to a small one for a particular failure mode is another a big problem of risk analysis with FMEA. In FM8 and FM12, the difference between the CFRPNs and the MVRPNs shows that the proposed approach is more sensitive to the evaluation information of the experts, and it can reduce the duplication of RPNs, which is also an another problem needed to be handled in FMEA.

Above all, the propose approach in this paper is compatible with the one in the literature [3], which assures its effectiveness. And it is more sensitive to the evaluation information comes from experts, which means a promising application prospect for combine uncertain and imprecise information of experts in a FMEA group.

\section{Conclusions}

This paper contributes a new FMEA model to combine the imprecise evaluation information in a FMEA group. With the empty set in GET, according to the proposed FMEA model, the rating conflict from different experts' evaluation information is modeled properly. Compared with the model in the literature, the proposed approach is compatible and more sensitive to the assessment information, which assures it can be more accurate while combining the rating conflicts from different experts. The experiment results based on the case study of aircraft engine rotor blades verify the merits of the proposed approach.

\section{References}

[1] Natarajan, Dhanasekharan. Failure mode and effects analysis. Hazard Analysis Techniques for System Safety. John Wiley \& Sons, Inc., 2005:235-259.

[2] Chin, Kwai Sang, et al. "Failure mode and effects analysis using a group-based evidential reasoning approach." Computers \& Operations Research 36.6(2009):1768-1779.

[3] Yang, Jianping, et al. "Risk evaluation in failure mode and effects analysis of aircraft turbine rotor blades using Dempster-Shafer evidence theory under uncertainty." Engineering Failure Analysis 18.8(2011):2084-2092.

[4] Su, Xiaoyan, et al. "An improved method for risk evaluation in failure modes and effects analysis of aircraft engine rotor blades." Engineering Failure Analysis 26.12(2012):164-174.

[5] Manger, Ryan P., et al. "Failure mode and effects analysis and fault tree analysis of surface image guided cranial radiosurgery." Medical Physics42.5(2015):2449-2461.

[6] Sayler, Elaine, et al. "Clinical implementation and failure mode and effects analysis of HDR skin brachytherapy using Valencia and Leipzig surface applicators." Brachytherapy 14.2(2015):293-299.

[7] Feili, Hamid Reza, et al. "Risk analysis of geothermal power plants using Failure Modes and Effects Analysis (FMEA) technique." Energy Conversion \& Management 72.72(2013):69-76.

[8] A.J.J. Braaksma, W. Klingenberg, and J. Veldman. "Failure mode and effect analysis in asset maintenance: a multiple case study in the process industry." International Journal of Production Research51.4(2013):1055-1071.

[9] Liu, Hu Chen, L. Liu, and N. Liu. "Risk evaluation approaches in failure mode and effects analysis: A literature review." Expert Systems with Applications 40.2(2013):828-838.

[10] Du, Yuxian, et al. "A new method in failure mode and effects analysis based on evidential reasoning." International Journal of System Assurance Engineering \& Management 5.1(2014):1-10.

[11] Bozdag, Erhan, et al. "Risk Prioritization in Failure Mode and Effects Analysis Using Interval Type-2 Fuzzy Sets." Expert Systems with Applications 42.8(2015):4000-4015. 
[12] Pillay, A., and J. Wang. "Modified failure mode and effects analysis using approximate reasoning." Reliability Engineering \& System Safetyvolume 79.1(2003):69-85(17).

[13] Du, Yuxian, et al. "New Failure Mode and Effects Analysis: An Evidential Downscaling Method." Quality \& Reliability Engineering International (2014).

[14] Liu, Hu Chen, et al. "Failure mode and effects analysis using D numbers and grey relational projection method." Expert Systems with Applications41.10(2014):4670-4679.

[15] Dempster, Arthur P. "Upper and Lower Probabilities Induced by a Multi-Valued Mapping." Annals of Mathematical Statistics 38.2(1967):325-339.

[16] Shafer G. A Mathematical Theory of Evidence. Princeton University Press, (1976), Princeton.

[17] Shafer, Glenn. "A mathematical theory of evidence." Technometrics 20.1(1978):242.

[18] Deng, Yong. "Generalized Evidence Theory." Applied Intelligence 43.3(2014):1-14.

[19] Deng, Yong, W. Jiang, and D. Han. "Basic Frame of Generalized Evidence Theory." Journal of Xian Jiaotong University 44.12(2010):119-124.

[20] Deng, Yong, and D. Han. "Methods to Determine Generalized Basic Probability Assignment in Generalized Evidence Theory." Journal of Xian Jiaotong University (2011).

[21] Yong Deng. "D Numbers: Theory and Applications." Journal of Information \& Computational Science 9: 9 (2012) 2421-2428

[22] Deng, Xinyang, et al. "Supplier selection using AHP methodology extended by D numbers." Expert Systems with Applications41.1(2014):156-167.

[23] Sankar, Nune Ravi, and B. S. Prabhu. "Modified approach for prioritization of failures in a system failure mode and effects analysis."International Journal of Quality \& Reliability

Management 18.18(1984):324-336. 